\title{
An evaluation of Universal Sample Processing method under Revised National Tuberculosis Control Programme set up for the diagnosis of pulmonary tuberculosis
}

Savitha Parashuram, Subba Rama Prasad

Department of Microbiology, Sri Devaraj Urs Medical College, Tamaka, Kolar, Karnataka, India

Address for the Correspondence: Dr. S. R. Prasad, Department of Microbiology, Sri Devaraj Urs Medical College, Tamaka, Kolar - 563 101, Karnataka, India.

E-mail: subbaramaprasad@ gmail.com

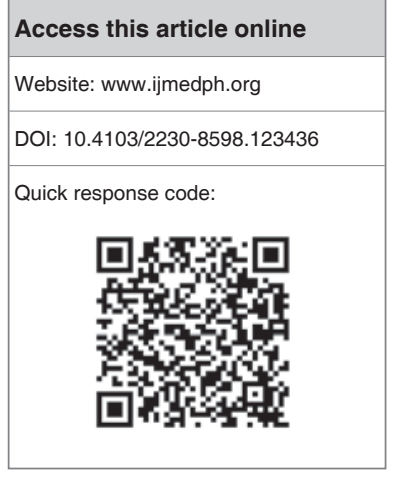

Context: The main stay in the management of tuberculosis is its early diagnosis and treatment. Aim: To evaluate the utility of Universal Sample Processing (USP) method, claimed to be more sensitive than direct smear microscopy. Materials and Methods: Sputum samples from 1000 patients with history of cough for more than two weeks, visiting the two RNTCP centers between November 2011 and November 2012, were subjected to direct Ziehl-Neelsen staining and staining after USP method. To find out if there was any difference in the time taken to detect Acid Fast Bacilli (AFB) between the methods, the smears were screened by two trained RNTCP technicians. Sputum samples positive for AFB in the USP method were put up for culture on Lowenstein-Jensen medium to evaluate the suitability of USP method as a pre-treatment procedure before culture. The growth was identified as Mycobacterium tuberculosis (MTB) by the Niacin test. Results: Ninety four samples were positive for AFB by both methods. The USP method did not detect more positives among those which were found negative by direct microscopy. There was no significant difference in the time taken to detect AFB between the methods. Among the 94 smear positive samples subjected to USP method as a pre-treatment procedure, only $40(42.5 \%)$ samples yielded growth of MTB. Conclusions: USP technique did not offer additional advantage over direct microscopy for detection of AFB to diagnose pulmonary tuberculosis. As a pre-treatment procedure, USP method yielded low mycobacterial growth even from those samples positive for AFB by microscopy, making it unsuitable for culture of MTB.

Key words: Acid Fast Bacilli, pulmonary tuberculosis, sputum microscopy, Universal Sample Processing (USP) method

\section{INTRODUCTION}

Tuberculosis continues to be a major public health problem in India and other developing countries. ${ }^{[1]}$ The emphasis in the control of tuberculosis is on early detection and prompt treatment. In India it is carried out under the auspices of the Revised National Tuberculosis Control Programme (RNTCP).

Under RNTCP, even today, in most laboratories sputum microscopy after Ziehl-Neelsen (ZN) staining remains the main stay to detect the Acid Fast Bacilli (AFB) to diagnose tuberculosis. Microscopy after $\mathrm{ZN}$ staining is easy to perform, cost effective, and is highly specific for tuberculosis in countries like India, where tuberculosis is rampant. However, sputum microscopy after ZN staining has a poor sensitivity; it detects AFB only when the bacilli are found at a concentration of 10,000 bacilli per mL of the sample..$^{[2]}$

In recent years, newer methods to increase the sensitivity of sputum microscopy have been explored and some are promising. ${ }^{[3]}$ These methods employ physical and chemical methods to process the sputum before staining and microscopic examination. Many chemicals, which liquefy the sputum, such as Sodium hydroxide and N-acetyl L-cysteine solution, sodium hypochlorite (bleach), C18-carboxy propyl betaine, chitin, and Xylol have been used. The sputum samples thus treated with chemicals have 
been subjected to a concentration procedure such as centrifugation, sedimentation, or floatation before making smears, staining, and finally microscopic examination. ${ }^{[3]}$

One such method, smear microscopy after Universal Sample Processing (USP) has been reported to detect AFB in concentrations as low as 250-300 bacilli per $\mathrm{mL}$ of sample with a sensitivity of 98\% and a specificity of $91 \% \cdot{ }^{[4]}$ This method employs a cocktail of chemicals: Guanidinium hydrochloride, Tris-Cl, EDTA, Sarkosyl, Beta mercaptoethanol, to pretreat the sputum before it is concentrated by centrifugation. However the claims of high sensitivity and specificity made with regard to this test need to be substantiated by other studies. ${ }^{[5]}$ Here we report the findings of our study to evaluate the utility of ZN staining after USP technique on the sputum samples collected from two Designated Microscopic Centers (DMC) of RNTCP: One situated at the District hospital and the other at our tertiary care center in the same district. In addition, as USP method has also been reported to be suitable for processing sputum samples as a pre-treatment procedure for AFB culture, we have evaluated this aspect by subjecting the sputum samples positive for AFB to pre-treatment with USP method before culture.

\section{MATERIALS AND METHODS}

Well coughed up sputum samples (one collected on spot and another early in the morning) collected from 1000 consecutive patients visiting the two RNTCP screening centers between November 2011 and November 2012 were included in the study. All these patients had cough for more than two weeks duration. Among the samples processed, 704 (70.4\%) were from patients who visited the RNTCP centre situated at the government district hospital, and 296 (29.6\%) samples from the tertiary care teaching hospital. The patients who were on anti-tubercular drugs were excluded.

The sputum samples collected were subjected to direct smear microscopy as per the RNTCP guidelines as well as USP smear microscopy in parallel. ${ }^{[4,6]}$ The smears positive for AFB in the direct smear microscopy were graded as per RNTCP guidelines into $1+$, $2+$, and $3+{ }^{[6]}$

The USP smear microscopy was performed as per the method described by Chakravorty et al. ${ }^{[4,7]}$ In brief, the sputum samples were mixed with 2-3 volumes of the USP solution, which contained the following chemicals: 4-6 M of guanidinium hydrochloride (a chaotropic agent which disrupts the hydrogen bonds), $50 \mathrm{mM}$ Tris chloride (pH 7.5), 25 mM Ethylene Diamine Tetra Acetic acid (EDTA), 0.5\% sarkosyl, and 0.1-0.2 M Beta mercaptoethanol. These ingredients together brought about mucolysis and acted as detergents. Thus by their action the chemicals used lysed all the cells in the sputum sample except the tubercle bacilli. Then the samples were homogenized by vortexing or shaking by hand, incubated for $15 \mathrm{~min}$ at room temperature. To the homogenate, thus obtained, $5-15 \mathrm{ml}$ of sterile water was added and centrifuged at $9000 \times \mathrm{g}$ for $20 \mathrm{~min}$. The sediment formed was mixed well and a part (10\%) of the sediment was used for making the smear and a part of it was used for culture. The smear was air dried, subjected to ZN staining and observed under the microscope. ${ }^{[6]}$

To detect whether there was any difference in the time taken to detect the AFB between direct smear microscopy and USP smear microscopy, 24 slides positive for AFB (8 slides of each grade) were screened by two trained RNTCP technicians and the time taken was noted.

The samples positive for AFB after USP method were cultured on LJ medium to find out the yield. The growth on the LJ medium was confirmed as Mycobacterium tuberculosis (MTB) by the Niacin test.

\section{RESULTS}

Among the patients from whom sputum samples were tested, 641 $(64.1 \%)$ were males and $359(35.9 \%)$ were females; $940(98.2 \%)$ of them were above 20 years of age.

Table 1 presents the sputum samples positive for AFB by direct smear microscopy and USP smear microscopy method according to the centers from which they were collected.

Of the 1000 sputum samples processed, 94 (9.4\%) samples were positive for AFB by both direct and USP smear microscopy. There was no difference in the rate of positives detected by direct smear microscopy and USP smear microscopy, among the samples tested. There were no extra positives detected by USP smear microscopy among those samples which were negative by direct smear microscopy.

The microscopic picture seen after direct smear microscopy differed substantially from that seen after USP method.

Table 2 presents the grading of the positive samples for AFB in direct smear microscopy according to RNTCP guidelines and the number detected to be positive by the two methods.

Among the 94 sputum samples positive for AFB, most of them belonged to grades $1+, 2+$, and $3+$. There was no difference in the number of positives detected between USP smear microscopy and direct smear microscopy in each of the grades.

Classically as described, the direct smear microscopy showed AFB distributed in singles or small groups of two or three on thick, heavily counterstained blue background with numerous pus

\begin{tabular}{|c|c|c|c|}
\hline RNTCP centers & $\begin{array}{c}\text { Total number } \\
\text { of samples } \\
\text { tested }\end{array}$ & $\begin{array}{l}\text { Positives by } \\
\text { direct smear } \\
\text { microscopy }\end{array}$ & $\begin{array}{l}\text { Positives by } \\
\text { USP smear } \\
\text { microscopy }\end{array}$ \\
\hline District hospital & 704 & $65(9.2 \%)$ & $65(9.2 \%)$ \\
\hline $\begin{array}{l}\text { Tertiary care } \\
\text { teaching hospital }\end{array}$ & 296 & $29(9.7 \%)$ & $29(9.7 \%)$ \\
\hline Total & 1000 & $94(9.4 \%)$ & $94(9.4 \%)$ \\
\hline
\end{tabular}


cells, macrophages, and lymphocytes. The USP smear microscopy [Figure-1] showed a clear background without any cells and cellular debris and the bacilli were seen clumped together at one or more sites. The stained smear after USP method could not be graded as the bacilli were seen clumped together at one or more sites.

Table 3 presents the mean time taken to detect AFB between direct smear microscopy and USP smear microscopy by the trained RNTCP technicians.

There was no statistically significant difference in the time taken to detect the AFB in stained direct smear microscopy and USP smear microscopy $(P=0.7236$, at $95 \% \mathrm{CI})$.

Of the 94 sputum samples positive for AFB inoculated on to LJ medium, only 40 (42.5\%) samples showed mycobacterial growth on LJ medium, which was confirmed by niacin test; 31 (32.9\%) showed no growth and $23(24.46 \%)$ samples inoculated grew contaminants. The mean time taken for mycobacterial growth on LJ medium was 22 days.

\section{DISCUSSION}

We report the results of an incremental yield study which compares the positivity rate obtained by direct smear microscopy with that obtained by USP method. Of the 1000 sputum samples processed from consequent patients suspected of tuberculosis attending two RNTCP centers, 94 (9.4\%) samples were positive for AFB by ZN staining in both direct smear microscopy and microscopy after USP processing. The specimens which were positive by direct smear microscopy were also positive for AFB after USP smear microscopy. Since, the sensitivity of direct sputum microscopy is low, it was expected to miss AFB in some of the samples; accordingly USP smear microscopy was expected to detect at least some of these as it was claimed to have a high sensitivity. ${ }^{[4]}$ But, in our study, none of the samples which was negative by direct smear microscopy was positive for AFB after USP smear microscopy. Thus there was no improvement in the AFB detection rate after subjecting sputum samples to USP method over that detected by direct sputum microscopy.

Chakravorty et al. reported that USP smear microscopy showed a sensitivity and specificity of $98.2 \%$ and $91.4 \%$, respectively, compared to $68.6 \%$ and $92.6 \%$, by direct smear microscopy taking culture as gold standard. ${ }^{[4,7]}$ In addition, they also detected 100 (30.48\%) samples, which were negative by direct smear microscopy, as positive for AFB after USP smear microscopy. ${ }^{\left[{ }^{4]}\right.}$ Thus our findings contrast with the findings reported by Chakravorty et al. ${ }^{[4]}$ Our findings concur with the findings of a study by Daley et al., who found that USP method was not significantly more sensitive when compared with direct smear microscopy. ${ }^{[8]}$

Cattamanchi et al. in Uganda showed that the USP method did not significantly improve the overall diagnostic performance of smear microscopy when N-acetyl-L-Cysteine Sodium hydroxide solution method was compared with the USP method. ${ }^{[5]}$ The lower detection

\begin{tabular}{lcc}
$\begin{array}{l}\text { Table 2: The positives detected by USP method } \\
\text { according to the grading done by direct smear } \\
\text { microscopy }\end{array}$ \\
$\begin{array}{l}\text { Grading for AFB } \\
\text { by direct smear } \\
\text { microscopy }\end{array}$ & $\begin{array}{c}\text { Number of samples } \\
\text { detected as positive } \\
\text { by direct smear } \\
\text { microscopy (\%) }\end{array}$ & $\begin{array}{c}\text { Number of positive } \\
\text { samples in USP } \\
\text { smear microscopy } \\
\text { (\%) falling under } \\
\text { the different grades } \\
\text { as per direct smear } \\
\text { microscopy }\end{array}$ \\
\hline $\begin{array}{l}\text { Scanty } \\
1+\end{array}$ & $12(12.7)$ & $\begin{array}{c}12(12.7) \\
24(25.53)\end{array}$ \\
$\begin{array}{l}\text { 3+ } \\
\text { Total number of }\end{array}$ & $28(29.7)$ & $28(29.7)$ \\
positives & $30(31.9)$ & $30(31.9)$ \\
\hline
\end{tabular}

\begin{tabular}{|c|c|c|c|}
\hline Grade & $\begin{array}{c}\text { Time taken to } \\
\text { detect AFB in Direct } \\
\text { smear microscopy } \\
\text { in seconds }\end{array}$ & $\begin{array}{l}\text { Time taken to detect } \\
\text { AFB after USP } \\
\text { smear microscopy in } \\
\text { seconds }\end{array}$ & $\begin{array}{c}P \text { value at } \\
95 \% \mathrm{Cl}\end{array}$ \\
\hline $1+$ & 43.44 & 42.94 & 0.8769 \\
\hline $2+$ & 28.38 & 26.94 & 0.1698 \\
\hline $3+$ & 14.00 & 12.19 & 0.1840 \\
\hline Total & 28.40 & 27.40 & 0.7236 \\
\hline
\end{tabular}

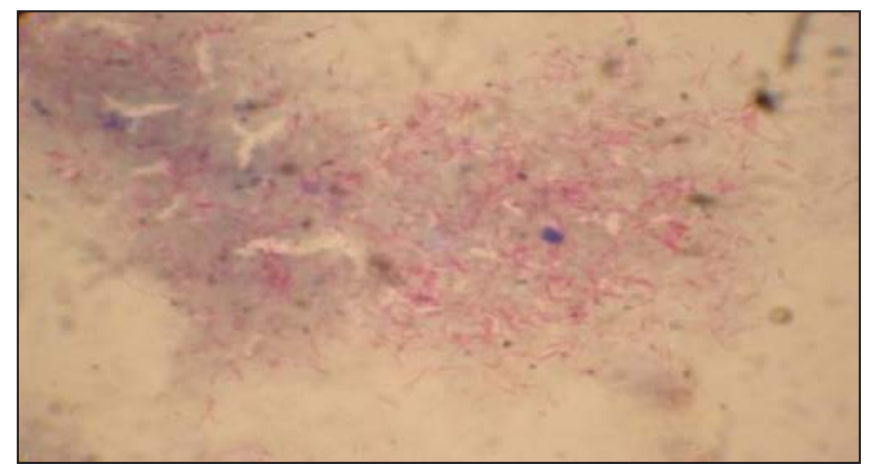

Figure:1 Microscopic picture of USP smear after ZN staining

rate by the USP method in their study was attributed to the lesser centrifugation speed of $3000 \times \mathrm{g}$ used by them, compared to that of $6000 \mathrm{~g}$ used by original authors. ${ }^{[9]}$ However we used a speed of $9000 \times g$ for $20 \mathrm{~min}$ to centrifuge the samples after treatment with USP fluid, as the smear and culture positivity has been shown to consistently improve at this speed. ${ }^{[10]}$ In spite of this modification, there was no improvement in the detection rate of USP method in our hands.

The time taken for the detection of AFB by two different trained RNTCP technicians from the hospitals where the study was conducted did not show any significant difference $(P=0.7236)$ in the speed of detection of AFB in direct smear microscopy and USP smear microscopy. Thus USP smear microscopy did not have 
any advantage over direct microscopy with respect to the speed of detection.

Of the 94 samples positive for AFB by USP smear microscopy, 40 $(42.5 \%)$ samples grew MTB on LJ medium, no growth was seen in $31(32.9 \%)$ samples and $23(24.46 \%)$ samples grew contaminants. This lower recovery rate of mycobacteria from the smear positive sputum samples collected from patients who were not on treatment for tuberculosis, suggests that USP method may not be a suitable method for pre-treatment of samples before culture on LJ medium. It may be deleterious to mycobacteria. These observations made by us will not support the claims made by the original authors that USP method is suitable for both microscopy and culture.

Thus in our hands USP smear microscopy did not show any additional advantage over conventional direct smear microscopy to detect AFB in the diagnosis of pulmonary tuberculosis under RNTCP set up. Also mycobacterial culture after USP technique showed high contamination rates and low growth rate. With this experience we think that USP method may not find a place in the routine diagnosis of tuberculosis in developing countries.

\section{REFERENCES}

1. Park K. Epidemiology of communicable diseases. In: Park's text book of preventive and social medicine. $17^{\text {th }}$ ed. Jabalpur: BanarsidasBhanot; 2006. p.115-268.

2. Winn W Jr, Allen S, Janda W, Koneman E, Schreckenberger P, Woods G, Editor. Mycobacteria In; Koneman's colour Atlas and Text book of Diagnostic Microbiology. $6^{\text {th }}$ ed. Philadelphia; Lippincot Williams and
Wilkins; 1997. p. 983-9.

3. Steingart KR, Vivienne NG, Henry M, Hopewell CP, Ramsay A Cunningham J,etal. Sputum processing methods to improve the sensitivity of smear microscopy for Tuberculosis: A systematic review. Lancet Infect Dis 2006:6:664-74.

4. Chakravorty S, Dudeja M, Hanif M, Tyagi JS. Utility of USP methodology, combining smear microscopy, culture and PCR for diagnosis of Pulmonary Tuberculosis. J ClinMicrobiol 2005;43:2703-8.

5. Cattamanchi A, Davis JL, Worodria W, Yoo S, Matovu J,Kiidha J,et al. Poor performance of Universal Sample Processing method for diagnosis of pulmonary tuberculosis by smear microscopy and culture in Uganda J ClinMicrobiol 2008;46:3325-9.

6. Central TB Division, Directorate of Health Services, Ministry of Health and Family Welfare, New Delhi. Technical and Operational guidelines for TB control (Internet). 2005 (cited 2013 Nov 12). Available from: http://www health.bih.nic.in/Docs/Guidelines/Guidelines-TB-Control.pdf

7. Chakravorty S, Tyagi JS. Novel multipurpose methodology for detection of Mycobacteria in pulmonary and extra pulmonary specimens by smear microscopy, culture and PCR. J ClinMicrobiol 2005;43:2697-702.

8. Daley P, Michael JS,Kalaiselvan S, Latha A Mathai D, John KR, et al. A pilot study of short-duration sputum pretreatment procedures for optimizing smear microscopy for tuberculosis. PLOS One 2009:4:e5626.

9. Chakravorty S, Tyagi JS. Performance of modified Universal Sample Processing method in a field study in Uganda. J ClinMicrobiol 2009;47:517-8.

10. Perera J, Arachchi DM, The optimum relative centrifugal force and centrifugation time for improved sensitivity of smear and culture for detection of Mycobacterium tuberculosis from sputum. Trans Roy Soc Trop Med and Hyg 1999;93:405-9.

How to cite this article: Parashuram S, Prasad SR. An evaluation of Universal Sample Processing method under Revised National Tuberculosis Control Programme set up for the diagnosis of pulmonary tuberculosis. Int J Med Public Health 2013;3:250-3.

Source of Support: Nil, Conflict of Interest: None declared. 